\title{
Anti-proliferative Activity of Some Oleanolic Acid Derivatives with Potent Topoisomerase Inhibitory Activity on B16 Melanoma Cells
}

\author{
Ahmed Ashour, Ryuichiro Kondo and Kuniyoshi Shimizu
}

\begin{abstract}
Our previous study included the semisynthetic reactions on oleanolic acid, a common wood-derived oleanane-type triterpene. Ten rationally designed derivatives of oleanolic acid were synthesized based on docking studies and tested for their topoisomerase I and Il $\alpha$ inhibitory activity. Semisynthetic reactions targeted C-3, C-12, C-13 and C-17. Some of these compounds act as dual inhibitors for both topoisomerase I and Il $\alpha$ giving new anticancer agents. The cytotoxic activity of these compounds on B16 melanoma cancer cells was evaluated. Results showed that most of these compounds have a higher cytotoxic activity on B16 melanoma cells.
\end{abstract}

Keywords: oleanolic acid, topoisomerase I and II, inhibitor, cytotoxic activity, B16 melanoma cells.

\section{Introduction}

Molecular modeling systems provide powerful tools for building, visualizing, analyzing, and storing models of complex molecular systems that can help interpret structure activity relationship (Cohen et al. 1990) where the techniques of combinatorial chemistry have revolutionized the development of active chemical leads where currently, instead of medicinal chemists making derivatives from scratch, a procedure is used whereby syntheses are based on combinatorial processes so that modification can be made in iterative fashion (Newman 2008). Over the past several years there has been a steadily evolving use of molecular modeling "computer-assisted drug design" as a tool in drug design.

Inhibition of topoisomerases is one of the important mechanisms of anticancer drugs (Pommier 2013). Pentacyclic triterpenes such as boswellic, betulinic, ursolic and oleanolic acids are reported to inhibit topoisomerases I and Il $\alpha$ by competing with DNA for topoisomerase binding through direct interaction with the enzyme preventing topoisomerase-DNA complex formation in both topoisomerases. Structure activity relationship suggested the general pentacyclic ring structure as important system for topoisomerase inhibitory activity. Carboxylation of the pentacyclic ring structure was also suggested to be necessary for topoisomerase inhibition (Syrovets et al. 2000). Based on these consequences, oleanolic acid which has been isolated from the unused parts of Hibiscus sabdariffa (Amer et al. 2011) was suggested as backbone for rational design of specific topoisomerase inhibitors.

In our previous study (Ashour et al. 2014), we have synthesized ten derivatives of oleanolic acid (Fig. 1) and tested for their inhibitory activity against topoisomerase enzymes. It was found that some compounds act as a dual inhibitor for both topoisomerase I and II. In this study, we have tested the activity of these compounds on B16 cells as an example for the cancer cells in an attempt to find the mechanism by which these compounds can inhibit the proliferation of these cells.

\section{Materials and Methods}

\section{Plant Material}

Oleanolic acid was isolated and purified from a methanolic extract of unused parts of Hibiscus sabdariffa grown in Egypt (Amer et al. 2011).
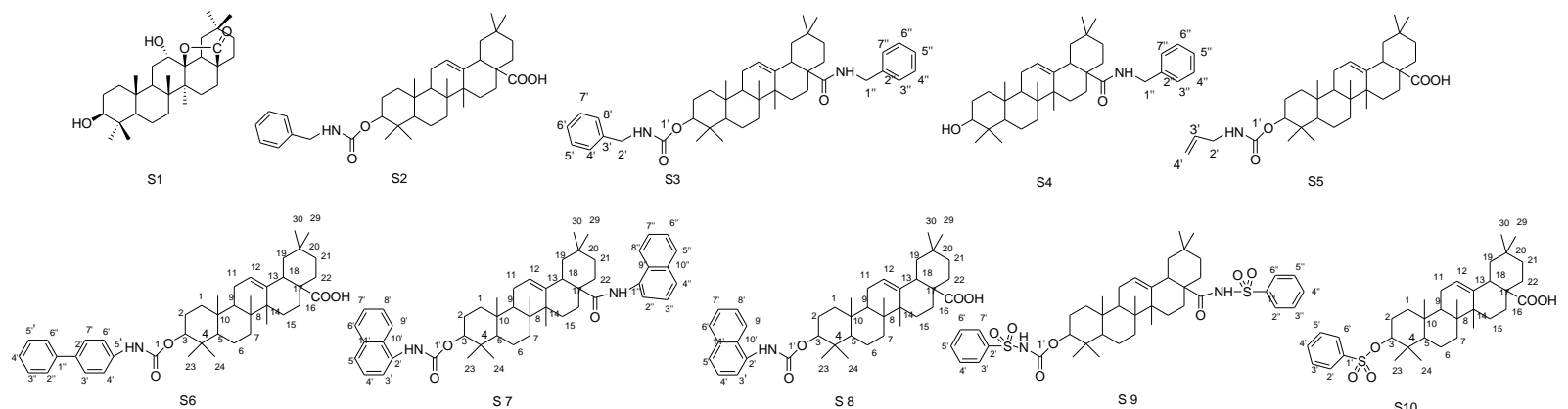

Figure 1. Structure of the synthesized compounds. 


\section{B16 Melanoma Cell Line Assay}

This assay was determined as described previously (Arung et al. 2007). The cells were placed in two plates of 24-well plastic culture plates (one plate for determining melanin and the other for cell viability) at a density of $1 \times 10^{5}$ cells/well and incubated for $24 \mathrm{~h}$ in media prior to being treated with the samples. After $24 \mathrm{~h}$, the media were replaced with $998 \mu \mathrm{L}$ of fresh media and $2 \mu \mathrm{L}$ of the test sample at maximum solubility $(n=3)$. At the same time, negative control ( $2 \mu \mathrm{L}$ DMSO) and positive control; Arbutin at concentration $50 \mathrm{mg} / \mathrm{mL}$ in DMSO were tested. The cells were incubated for an additional $48 \mathrm{~h}$, and then the medium was replaced with fresh medium containing each sample. After $24 \mathrm{~h}$, the remaining adherent cells were assayed. To determine the melanin content (for one plate) after removing the medium and washing the cells with PBS, the cell pellet was dissolved in $1.0 \mathrm{~mL}$ of $1 \mathrm{~N} \mathrm{NaOH}$. After overnight keeping in dark, the crude cell extracts were assayed by using a microplate reader at $405 \mathrm{~nm}$ to determine the melanin content. The results from the cells treated with the test samples were analyzed as a percentage of the results from the control culture. On the other hand, cell viability was determined by using MTT assay which provides a quantitative measure of the number of viable cells by determining the amount of formazan crystals produced by metabolic activity in treated versus control cells. So, for the other well plate, $50 \mu \mathrm{L}$ of MTT reagent in PBS $(5 \mathrm{mg} / \mathrm{mL})$ was added to each well. The plates were incubated in a humidified atmosphere of $5 \%$ of $\mathrm{CO}_{2}$ at $37^{\circ} \mathrm{C}$ for $4 \mathrm{~h}$. After the medium was removed, $1.0 \mathrm{~mL}$ isopropyl alcohol (containing $0.04 \mathrm{~N} \mathrm{HCl}$ ) was added, and the absorbance was measured at $570 \mathrm{~nm}$ after overnight keeping in dark.

\section{Results and Discussion}

\section{B16 Melanoma Cell Biological Activity}

B16 melanoma cells have been known to produce melanin pigment. To understand the mode of action of oleanolic acid derivatives against cells, their effects on melanin production and cell growth of B16 melanoma cells have been evaluated. From the results of B16 melanoma cell assay (Fig. 2), it was found that Compounds S5, S9 and S10 have a high cytotoxic activity. These compounds may inhibit the growth of the cancer cells through inhibition of topoisomerase enzyme as we have reported the high inhibitory activity of these compounds against topoisomerases in our previous study (Ashour et al. 2014) or through their cytotoxic effect caused by other mechanisms. However we have also found that compounds S1, S3, S4 and S7 also have good melanin biosynthesis inhibitory activity in B16 melanoma cells with less cytotoxic activity. It means these compounds (S1, S3, S4 and S7) inhibit the function of the cancer cells such as melanin biosynthesis by a mechanism other than the cytotoxicity. The investigation for clarifying the precise mode of action of them are under investigation.

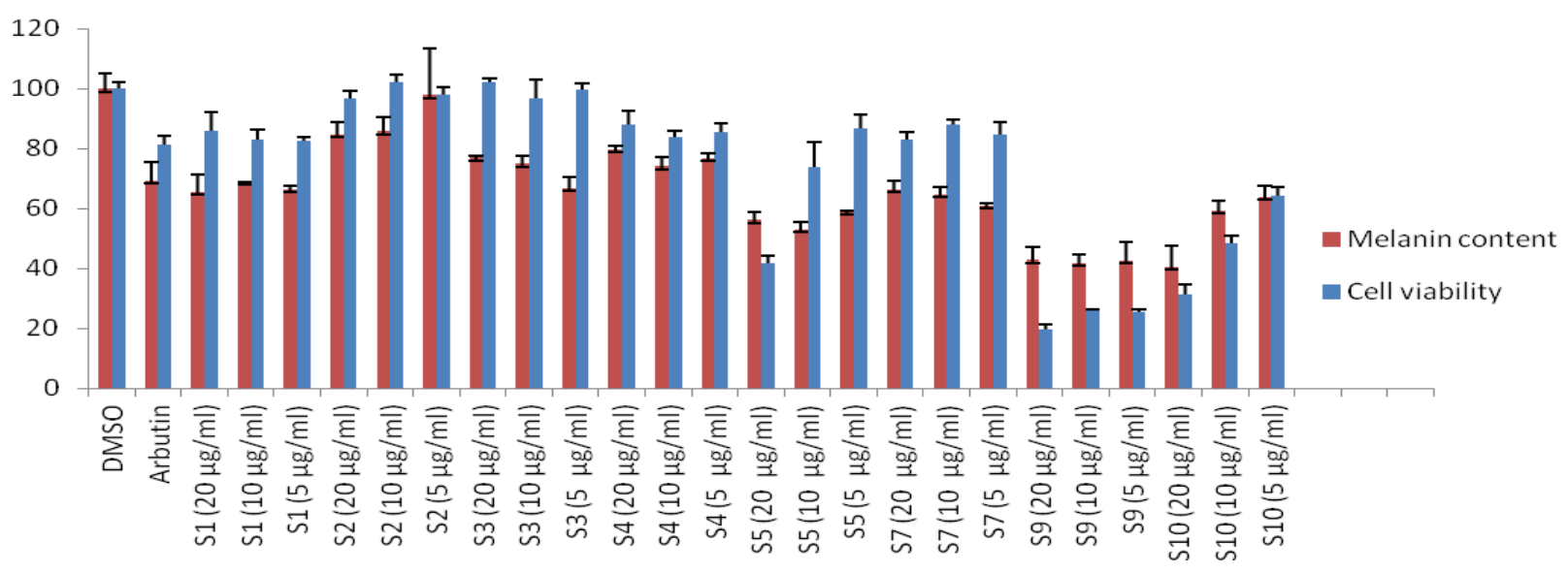

Figure 2. Effect of compounds on melanin formation in B16 melanoma cell. The values are represented as the mean \pm standard deviation $(S D), n=3$. Significant difference from the control value and each compound was determined by Student's t-test: ${ }^{*} P<0.05,{ }^{* *} P<0.01$. 


\section{Reference}

Amer, M.; S. El-Sharkawy; A. Marzouk; and A. Ashour. 2011. Chemical Constituents of Corncobs. J. of Environ. Sci. 40: 251-258.

Arung, E.; K. Shimizu; and R. Kondo. 2007. Structure Activity Relationship of Phenyl Substituted Polyphenols from Artocarpus heterophyllus as Inhibitors of Melanin Biosynthesis in Cultured Melanoma Cell. Chemistry \& Biodiversity 4: 21662171.

Ashour, A.; S. El-Sharkawy; M. Amer; F. Abdel Bar; Y. Katakura; T. Miyamoto; N. Toyota; T. Hai Bang; R. Kondo; and K. Shimizu. 2014. Rational Design and Synthesis of Topoisomerase I and II Inhibitors Based on Oleanolic Acid Moiety for New Anticancer Drugs. Bioorganic and Medicinal Chemistry 22(1): 211-220.

Cohen, N.; J. Blaney; C. Humblet; P. Gund; D. Barry. 1990. Molecular Modeling Software and Methods for Medicinal Chemistry. J. Med. Chem. 33: 584-594.

Newman, D. 2008. Natural Products as Leads to Potential Drugs: An Old Process or the New Hope for Drug Discovery. J. Med. Chem. 51: 2589-2599.
Pommier, Y. 2013. Drugging Topoisomerases: Lessons and Challenges. ACS Chemical Biology 8: 82-95.

Syrovets, T.; B. Büchele; E. Gedig; R. Slupsky; T. Simmet. 2000. Acetyl-Boswellic acids are Novel Catalytic Inhibitors of Human Topoisomerases I and Ila. Mol. Pharmacol. 58: 71-81.

\footnotetext{
Ahmed Ashour

Department of Pharmacognosy, Faculty of Pharmacy,

Mansoura University, Mansoura, Egypt

Tel : 002-01001737941

E-mail : ahmedadelashour@yahoo.com

Ryuichiro Kondo

Department of Agro-environmental Sciences,

Faculty of Agriculture, Kyushu University, Fukuoka, Japan

E-mail : ryukondo@agr.kyushu-u.ac.jp

Kuniyoshi Shimizu

Department of Agro-environmental Sciences,

Faculty of Agriculture, Kyushu University, Fukuoka, Japan

Tel/Fax : 092-642-3002

E-mail : shimizu@agr.kyushu-u.ac.jp
} 